\title{
Article \\ Comprehensive Evaluation Method of Ethnic Costume Color Based on K-Means Clustering Method
}

\author{
Linqi Zhao ${ }^{1}$, Zhenya Wang ${ }^{1, *}$, Yaxue Zuo ${ }^{2}$ and Danyang $\mathrm{Hu}^{1}$ \\ 1 School of Mechanical Engineering, Shandong University, Jinan 250061, China; \\ 202014434@mail.sdu.edu.cn (L.Z.); 202114417@mail.sdu.edu.cn (D.H.) \\ 2 School of Design, Shanghai Jiaotong University, Shanghai 200240, China; zuoyaxue@mail.sdu.edu.cn \\ * Correspondence: wangzhenya@sdu.edu.cn
}

check for

updates

Citation: Zhao, L.; Wang, Z.; Zuo, Y.; $\mathrm{Hu}, \mathrm{D}$. Comprehensive Evaluation Method of Ethnic Costume Color Based on K-Means Clustering Method. Symmetry 2021, 13, 1822. https://doi.org/10.3390/ sym13101822

Academic Editors: José Carlos R. Alcantud and Juan Luis García Guirao

Received: 25 May 2021

Accepted: 25 September 2021

Published: 30 September 2021

Publisher's Note: MDPI stays neutral with regard to jurisdictional claims in published maps and institutional affiliations.

Copyright: (c) 2021 by the authors. Licensee MDPI, Basel, Switzerland. This article is an open access article distributed under the terms and conditions of the Creative Commons Attribution (CC BY) license (https:// creativecommons.org/licenses/by/ $4.0 /)$.

\begin{abstract}
Color is the external manifestation of ethnic minority culture, and the costume of each ethnic group has its objective color matching rules. In the color design of minority costumes, there is often a lack of scientific evaluation methods. Aiming at this problem, this article proposed a comprehensive evaluation method, based on the K-Means clustering method, for evaluating color matching schemes of minority costumes. We used the K-Means clustering method to analyze the objective laws of minority costume colors, and based on the objective laws found, we extracted the objective evaluation indicators. With the AHP (analytic hierarchy process) method, the judgment matrix was established to obtain the relative weights of each cultural image and objective evaluation indicator. Based on the trapezoidal fuzzy number, the user's evaluation value of the cultural image index was clarified. The GRA-TOPSIS evaluation method was introduced to rank the color matching schemes of minority costumes. Taking the evaluation of the color matching scheme of Yi costumes as an example, this article confirmed that the proposed comprehensive evaluation method can effectively screen out the color matching schemes with the characteristics of minority costumes and can rank the color schemes to be evaluated according to their relative similarity degree to the color characteristics of minority costumes. The method integrated subjective and objective evaluations, overcame the problem of contradictory results of subjective and objective evaluations, and achieved a certain degree of symmetry between the objectivity of the color laws of minority costumes and the subjectivity of the cultural image of minority costumes. In addition, we also found the possibility of using K-Means clustering to extract the main color features of minority costumes to improve the design of color schemes.
\end{abstract}

Keywords: ethnic costume; color matching schemes; K-Means clustering; GRA-TOPSIS; comprehensive evaluation

\section{Introduction}

Minority costumes have distinct regional, folklore, and cultural characteristics. In addition, the minority costume is an important symbol of ethnic group identification and the precious wealth of the Chinese nation [1]. As time passed, they have gradually formed their own distinctive color system, which has become an important carrier for beautifying life and displaying national characteristics. To decorate oneself with color is the primitive instinct and impulse of mankind [2]. As a commodity, the visual appearance and color of minority clothing are the most important factors for consumers [3]. When choosing a product, consumers often make decisions within $90 \mathrm{~s}$, and the color of the product is the most important factor influencing the consumer's decision [4]. Therefore, when carrying out the innovative design of minority costumes, designers should devote more energy to the aspect of color design.

Evaluation is an indispensable part of the costume color design process, and evaluation errors are likely to lead to the failure of design. Evaluating the costume color of an ethnic minority group is conductive to its innovative design and the development of related 
products with ethnic minority characteristics, so that ethnic minority products can better enter the mass market and serve more consumers. Product color image evaluation mainly uses various evaluation methods to seek objective, fair, and effective evaluation results [5], including the evaluation method based on Kansei engineering, color harmony theory, gray correlation theory, or mathematical methods, etc. Wang et al. [6] proposed a compensatory evaluation method for color scheme based on eye movement tracking technology and conducted an objective quantitative evaluation for color schemes. Based on the fuzzy-set theory, Chen et al. [7] used the Kansei engineering model to evaluate the emotional image of the color pattern design in aircraft cockpits. Bai and Xue [8] analyzed the three attributes of hue, brightness, and saturation of fashion color samples and then independently designed the color matching schemes based on the fashion color schemes. Zhang et al. [9] used the gray correlation theory combined with the Kansei engineering model to mine the macro and microscopic factors in product color brand image, improving the accuracy of the correlation mining between product color design elements and brand image. He et al. [10] proposed a product color design and evaluation method based on color harmony theory, which can effectively analyze the relationship between beauty and cultural image. Yuan et al. [11] used Kansei engineering and the interactive genetic algorithm to obtain the satisfactory color design scheme. Lu et al. [12] used the semantic difference method of Kansei engineering to make perceptual evaluation on the clothing color scheme. In addition, according to the subjective impression generated by different colors of upper and lower garments, they proposed suggestions for female consumers to choose clothing color schemes when attending different occasions. Yang et al. [13] proposed a product color design method based on color cases and gray correlation analysis. They carried out color screening on product color samples, used color case method to represent color samples and established a mapping mechanism, and the gray correlation degree was calculated to analyze the image correlation between product color design schemes before and after mapping. Yang et al. [14] analyzed the orientation of the color image of the excavator and the development trend of the excavator color, based on which a mathematical model of Dunn's correlation degree was established through mathematical analysis. Then, they selected the optimal scheme through numerical analysis. The above studies either focus on the user's perceptual evaluation or focus on the degree of conformity between evaluation objects and objective laws, but there are few studies focusing on the combination of both subjective and objective evaluation. Regarding the research on the colors of ethnic minority costumes, related scholars have conducted research from the dimension of culture, geographical environment, cognition, and symbolism, but there are few discussions on design evaluation.

With the development of color technology, there have been relatively mature methods for quantitative analysis of color. In recent years, such methods have been used in clothing research. Jiang et al. [15] collected 480 colors of solid shirts from different brands and used the mean shift clustering algorithm to classify them into 19 clusters in the CIELAB color space, analyzing their color perception and preference. Sharon Lin and Pat Hanrahan [16] presented a method for extracting color themes from images using a regression model trained on themes created by people. Tao et al. [17] selected the HSV color model, which is based on human vision, and proposed a method to identify the main color of clothing and quantify the hue, purity, and brightness, with which one can effectively calculate the clothing color. Xu et al. [18] converted the color of minority costume images to the HSV space, clustered the main color of each minority costume, and optimized the main color distribution ratio according to the color distance measurement to visually express the distribution of color feature values. The above studies focus on the analysis of objective laws, but less attention to the application of laws in design practice was paid.

Clustering analysis has been commonly used in almost all disciplines involving multivariate data analysis [19], and its main purpose is to search the sensible grouping(s) of a set of patterns, points, or objects [20]. In the field of color extraction, cluster analysis is also widely used. Traditional clustering algorithms, such as the fuzzy c-means (FCM) 
clustering technique, K-Means algorithm, hierarchical clustering algorithm [21-23], etc., and improved clustering algorithms, such as Student's t-based density peaks clustering, $\mathrm{K}$-hyperline clustering algorithm [24,25], etc., have been used in the researches of the quantitative analysis of image color. Among them, the K-Means clustering algorithm is a relatively simple color extraction method. The K-Means algorithm was discovered independently by Steinhaus, Lloyd, Ball and Hall, and MacQueen in different disciplines [26-29], and on this basis, a large number of scholars including Hatigan and Wong [30], Mladenovic [31], and Kalczynski [32] have improved the K-Means algorithm in different ways. The advantage of the K-Means algorithm is that the clustered pixels of the same kind are as compact as possible, and the various types are as separated as possible. The disadvantage is that it is very sensitive to noise, and too many iterations can cause the algorithm to run too long. On the one hand, the color extraction is based on the pixel color value of the image as the source data, which generally does not produce noise. On the other hand, the ethnic minority costumes are textiles, and their images are mostly composed of large blocks of the same color pixels, with relatively few continuous gradient colors [33], which makes the shortcomings of the K-Means clustering algorithm, such as too many iterations and too long running time, have little impact on the color processing results. Therefore, here we chose the K-Means clustering algorithm to extract the color values of ethnic minority costumes.

Through reading related literature, we found that color plays an important role in reflecting the aesthetic sensibilities of ethnic minorities, symbolizing their cultural connotations and conveying visual emotional semantics in ethnic minority costumes $[34,35]$. Therefore, color is a key factor in the design of ethnic minority costumes. Design evaluation refers to comparing the advantages and disadvantages of different solutions corresponding to the same design problem in the design process and then determining the ranking of the advantages and disadvantages of each solution, to find the best design solution. The significance of design evaluation is to correct design direction in time, ensure design quality, reduce design cost, and provide the basis for design improvement [36]. Considering the importance of color factor to ethnic minority costumes, it is necessary to use appropriate methods to evaluate the color matching schemes of ethnic minority costumes. However, to the best of our knowledge, there is almost no evaluation method for the color matching schemes of ethnic costumes.

The costume colors of an ethnic minority are closely related to their culture. The GRA-TOPSIS method is an evaluation method that integrates gray correlation analysis (GRA) and technique for order performance by similarity to ideal solution (TOPSIS). It is very suitable for calculating the matching degree between the color collocation of a minority costume and its cultural image.

The limitation of subjective evaluation is that it has defects, such as semantic differences and cognitive biases, while objective evaluation does not consider the consumer's cognitive feelings and is difficult to link with subjective evaluation [37]. K-Means clustering can quantitatively analyze the colors, which provides us with the possibility to conduct objective evaluation of the color matching schemes of minority costumes; GRA-TOPSIS can well reflect the matching degree of color collocation and subjective cultural intention of minority costumes, which is an ideal method for color evaluation of minority costumes. If we use the analytical hierarchy process to combine objective evaluation based on the K-Means clustering algorithm with subjective evaluation oriented by user perception, then we can put forward a comprehensive evaluation method for the color matching schemes of ethnic costumes. Therefore, on the basis of GRA-TOPSIS, in this article we use the K-Means clustering method to obtain objective laws of minority costume colors, which is the basis of the objective evaluation index, and combine it with the cultural image of minority costumes, which compose the subjective evaluation index, to form a comprehensive evaluation index system. Then, a comprehensive evaluation method for minority costume color matching schemes based on the K-Means clustering method is proposed.

Compared with the existing research, this article has the following innovations: 
(1) This article proposes a comprehensive evaluation method for minority costume color.

(2) This article obtains the objective law of minority costume and transforms it into the objective index in design evaluation, which finally achieves a certain degree of symmetry between the objectivity of minority costume color laws and subjectivity of cultural images of minority costume color.

(3) The color innovation design of minority costume is carried out and the proposed evaluation method is used to evaluate the design schemes, where the proposed method is proved effective.

The remainder of this article is structured as follows. Section two describes the KMeans clustering method, which is applied to the analysis of Yi costume color. In section three, the proposed comprehensive evaluation method is described. Section four applies the proposed method to evaluate the color of Yi costumes. In section five, conclusions are presented. The flow of our proposed comprehensive evaluation method is shown in Figure 1.

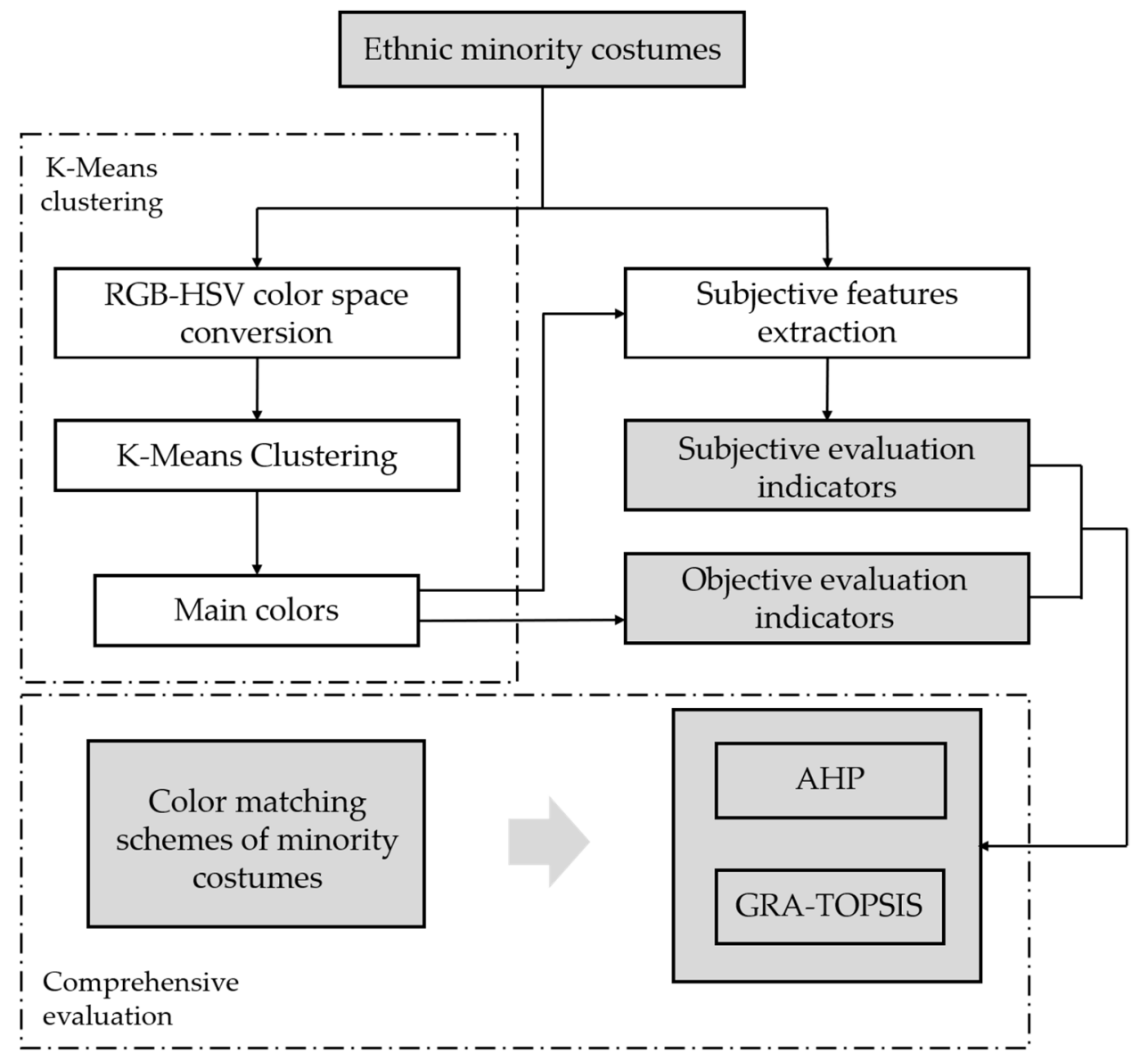

Figure 1. Flow of the proposed comprehensive evaluation method.

\section{K-Means Clustering}

In this article we take Yi costumes as an example, use the K-Means clustering method to extract the main components of its color, and further explore the objective law of these main components through statistical analysis.

\subsection{K-Means Clustering Method}

The K-Means clustering method is currently the most popular dynamic iterative clustering algorithm. The calculation criterion is based on the sum of squares of errors (SSE), and clustering is used as the Similarity index [38]. The specific algorithm steps of K-Means are: 
(1) Randomly select $k$ objects from the data as the initial cluster centers.

(2) Calculate the Euclidean distance of each cluster object to the cluster center and divide these cluster objects into the clusters corresponding to the closest cluster center according to the closest distance criterion.

(3) Calculate the average value of the data objects in each cluster center for the next iteration.

(4) Repeat step (2) and step (3) until the cluster center no longer changes or reaches the maximum number of iterations.

The formula for calculating the Euclidean distance from the data object to the cluster center is:

$$
\mathrm{d}\left(x, C_{i}\right)=\sqrt{\sum_{j=1}^{m}\left(x_{j}-C_{i j}\right)^{2}}
$$

In Equation (1), $x$ is the data object, $C_{i}$ is the $i_{t h}$ cluster center, $m$ is the dimension of data object, $x_{j}$ and $C_{i j}$ are the $j_{t h}$ attribute value of $x$ and $C_{i}$.

The formula for calculating the SSE of entire data is:

$$
S S E=\sum_{i=1}^{k} \sum_{x \in C_{i}}\left|\mathrm{~d}\left(x, C_{i}\right)\right|^{2}
$$

Among Equation (2), the value of SSE indicates whether the clustering result is good or not. The closer the SSE is to zero, the better the clustering result. Additionally, $k$ represents the number of clusters.

It is worth noting that the results of the K-Means clustering algorithm are sensitive to the location of initial clustering centers, so we adjusted the step 1) of the K-Means algorithm accordingly:

(1) Randomly and uniformly select one data object $c_{1}$ from the data $X$ as the initial cluster center.

(2) Calculate the Euclidean distance between data object $x$ and the nearest cluster center that has already been chosen, denoted by $D(x)$.

(3) Calculate the probability of each data object being selected as the next cluster center and select the data object corresponding to the maximum probability as the next cluster center.

(4) Repeat step (2) and step (3) until we have taken $\mathrm{k}$ centers altogether.

The formula for calculating the probability of each data object being selected as the next cluster center is:

$$
P(x)=\frac{D(x)^{2}}{\sum_{x \in X} D(x)^{2}}
$$

By making the above adjustment to the traditional K-Means algorithm, we can make the clusters as far away from each other as possible and reduce the error caused by the random selection of clustering centers in the traditional K-Means algorithm.

\subsection{RGB-HSV Color Space Conversion}

HSV color space is a color model oriented to visual perception, which is widely used in vision-based image processing tasks [39]. The HSV color model is a cylindrical representation of the standard RGB model [40]. Compared with RGB color space, its advantage lies in the separation of color information and brightness information. H stands for hue, which is related to wavelength and describes the hue attribute; $S$ stands for saturation, which represents the intensity of colors; V stands for value, which describes the brightness of colors. Human eyes are not equally sensitive to red, green, and blue, which are basic color components in the RGB color space. Among the monochromatic colors, human eyes are least sensitive to red and most sensitive to blue, so the RGB color space is a color space with poor uniformity. If the color similarity is directly measured by Euclidean distance, the result will have a large deviation from human vision. For a certain 
color, it is difficult to infer a more accurate value of the three RGB components to represent it. Therefore, RGB color space is suitable for display systems, but not for image processing. Since the lightness component is an independent factor of images, and the components of hue and saturation are so closely link with the pattern of human visual perception, this model can visually express the hue, vividness, and brightness of colors, which is convenient for color comparison. Therefore, it is easier to perform color segmentation in the HSV color space than in the RGB color space. The advantage of the HSV color space is suitable for the analysis of color laws of ethnic minority costumes.

The color sample images of minority costumes are in RGB mode and need to be converted to the HSV color space. The specific formulae are as follows [18]:

$$
\begin{gathered}
H=\left\{\begin{array}{c}
0, \text { if } \max =\min \\
60^{\circ} \times \frac{G-B}{\max -\min }, \text { if } \max =R \text { and } G \geq B \\
60^{\circ} \times \frac{G-B}{\max -\min }+360^{\circ}, \text { if } \max =R \text { and } G<B \\
60^{\circ} \times \frac{B-R}{\max -\min }+120^{\circ}, \text { if } \max =G \\
60^{\circ} \times \frac{R-G}{\max -\min }+240^{\circ}, \text { if } \max =B
\end{array}\right. \\
S=\left\{\begin{array}{c}
0 . \text { if } \max =0 \\
1-\frac{\min }{\max }+240^{\circ}, \text { if } \max \neq 0 \\
V=\max / 2.55
\end{array}\right.
\end{gathered}
$$

In the Equations (3)-(5), $R, G$, and $B$ correspond to the red, blue, and green channel values, respectively, and max and min respectively refer to the maximum and minimum values of $R, G$, and $B$, where, $H \in\left[0,360^{\circ}\right], S \in[0,100], V \in[0,100]$.

\subsection{Color Clustering of Yi Costume}

The Yi ethnic group is the most populous and widely distributed ethnic minority group in southwest China. There are many branches of its costumes, and they are colorful. Zhong and Zhou [41] selected some of the original Yi costumes from nearly a thousand sets of collections and compelled them into a book showing the appearance of Yi costumes, which are extremely representative. In order to make the research more representative, in this article we analyze a total of 52 pieces of costumes in the book as samples, as shown in Figure 2.
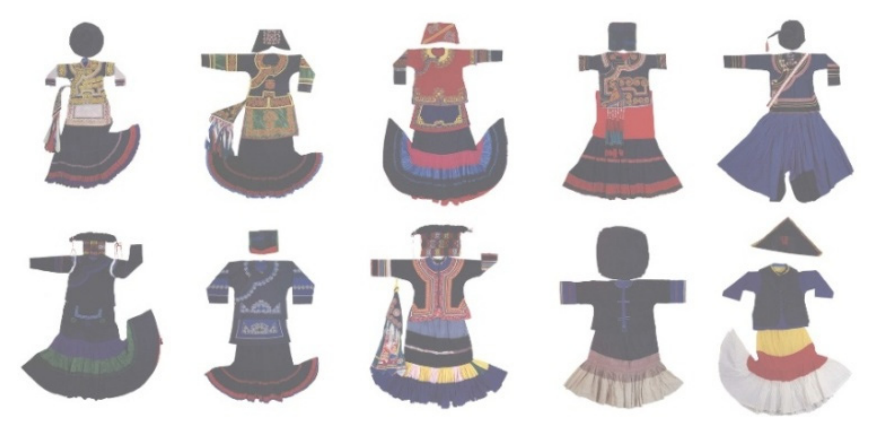

Figure 2. Partial experimental sample of Yi costumes.

In order to quantitatively analyze the Yi costume colors, we first performed K-Means clustering on each of the 52 samples to obtain the main colors of each sample costume.

First, we used Photoshop software to uniformly preprocess the sample costumes, removing the messy background that is irrelevant to the costumes, and unified the size of the sample images.

$\mathrm{K}$-value is the core parameter of K-Means clustering and its magnitude can be determined by the silhouette coefficient. The calculation of the silhouette coefficient $S$ is shown in Equation (7), where $a$ denotes the average distance between the sample point and all other points in the same cluster, i.e., the similarity of the sample point to other points in the 
same cluster; $b$ denotes the average distance between the sample point and all points in the next closest cluster, i.e., the similarity of the sample point to other points in the next closest cluster.

$$
S=\frac{b-a}{\max (a, b)}
$$

$S$ is the silhouette coefficient, which combines cohesiveness and separation, used to judge the performance of clustering. $S \in(-1,1)$, the lager the value $S$, the better the clustering result. What should be noted is that the $S$ here is distinguished from the $S$ used in HSV below.

By observing the color distribution of Yi costume samples, we find that Yi costumes are rich in colors and the number of colors varies for each sample. Therefore, we initially estimate $5 \leq k \leq 15$, and each sample costume color is clustered 11 times with different $k$-values, and the clustering results with the highest contour coefficient are taken as the final results. Next, we average all the points of each cluster center, and all the values obtained represent the main colors of each sample costume.

According to the above algorithm, a small program was written in Python. We input the sample RGB images, and the output results were main colors of the sample, the corresponding ratio, and HSV value of each main color.

We presented the main colors of all sample costumes in the HSV spatial coordinate system, as shown in Figure 3. Since we need to grasp the overall characteristics of Yi dress colors, we eliminated some main colors in the HSV spatial coordinate system that were obviously far from the group. Then, we made a block diagram of the remaining main colors corresponding to each sample according to the ratio and stitched the block diagrams corresponding to all samples into one diagram. Next, applying the same method as the first clustering, we perform a second clustering. The reason why we use the results of the first clustering for the second clustering is that we can compare the main colors obtained from the second clustering with the color rule of the Yi costumes recorded in the reference. If the main colors are consistent with the description in the literature, the result of the first clustering is reliable. If the primary color is inconsistent with the literature description, further research on the first clustering is required. The flowchart of the algorithm for the two clusterings is shown in Figure 4. Through the second clustering, we obtained the overall main color distribution of Yi costumes, as shown in Figure 5.

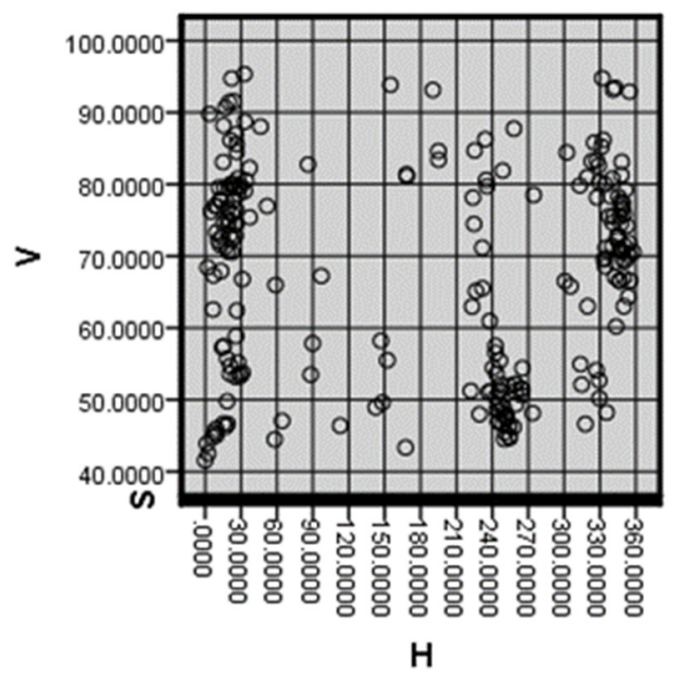

Figure 3. The main color distribution of the first clustering results under HSV space. 


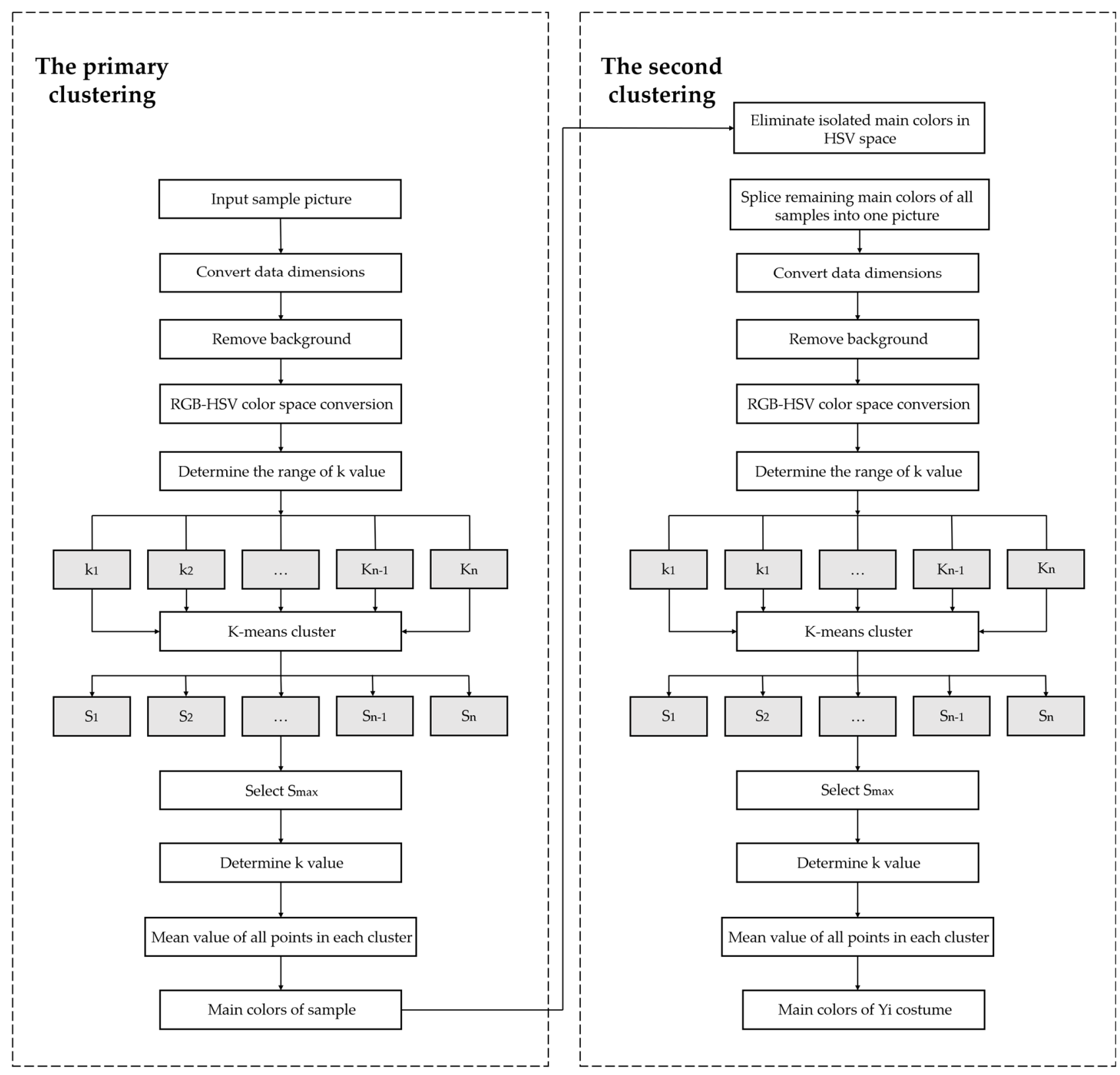

Figure 4. Flow chart of the algorithm for the two clusterings.

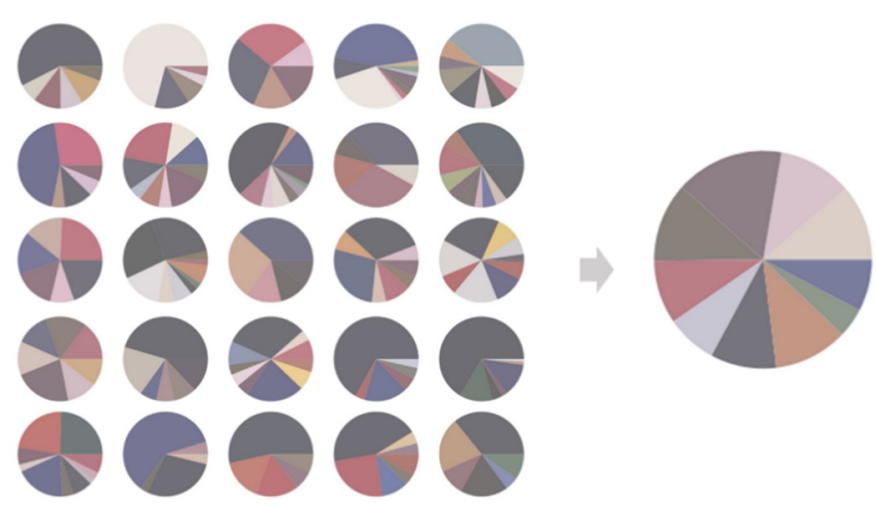

Figure 5. The primary and second clustering results. 


\subsection{Clustering Results and Analysis}

After sorting out the two clustering results, the main colors of each sample and the overall main colors of Yi costumes are obtained. From the perspective of color categories, among the main colors of Yi costumes obtained by the two rounds of clustering, the color distribution is relatively uniform, with the highest proportion of brown (HSV spatial coordinates: $328^{\circ}, 10.9 \%, 54.9 \%$ ) accounting for $16.11 \%$. The lowest color is green (HSV spatial coordinates: $130.909^{\circ}, 8 \%, 53.7 \%$ ), which accounts for $4.35 \%$. The remaining eight colors each account for between $7 \%$ and $12 \%$. Among them, navy blue accounted for $7.68 \%$, light gray accounted for $7.55 \%$, dark gray accounted for $9.79 \%$, warm gray accounted for $11.28 \%$, vermilion accounted for $9.45 \%$, pink accounted for $11.01 \%$, dark green accounted for $11.76 \%$, and dark yellow accounted for $11.01 \%$. They accounted for a mean value of $9.94 \%(\mathrm{SD}=1.52)$. The specific color distribution is shown in Table 1.

Table 1. Main colors of Yi costumes obtained by the secondary clustering.

\begin{tabular}{ccccccccccc}
\hline Color & & & & & & & & & \\
\hline H & 130.909 & 240 & 255 & 246 & 22.105 & 349.655 & 333 & 21.429 & 328 & 18.305 \\
S & 8 & 22.2 & 5.7 & 8.1 & 8.6 & 31.7 & 9.2 & 10.1 & 10.9 & 30.9 \\
V & 53.7 & 62 & 82.4 & 48.2 & 87.1 & 71.8 & 85.5 & 54.5 & 54.1 & 74.9 \\
Proportion & 4.35 & 7.68 & 7.55 & 9.79 & 11.28 & 9.45 & 11.01 & 11.76 & 16.11 & 11.01 \\
\hline
\end{tabular}

Similar to the traditional Chinese five-color view, the Yi ethnic group also has the term "five-color", which developed from the original "red, black and yellow" to the "square color", and then further developed. The "five-color" view of the Yi ethnic group is closely related to the Chinese traditional "five-color" view in terms of cultural meaning. To sum up the color symbols of the Yi ethnic group, black refers to black soil, red means fire, yellow means the sun, blue means the sky, and white means the earth [42].

After a long period of development, the contemporary Yi ethnic group has absorbed the color elements of other minority costumes on the basis of retaining the color characteristic of their traditional costumes. In addition to the five main colors of black, white, red, yellow, and blue, a small amount of green, pink, orange, cyan, and their similar colors have been added to the Yi people's color system. The distribution structure of the main colors of the second clustering result is consistent with the color distribution of Yi costumes recorded in the relevant literature.

From the perspective of hue, the results of the two rounds clustering show that the hues are concentrated in the color ring between $240^{\circ}$ and $30^{\circ}$, and we can see from the HSV color model, as shown in Figure 6, that the corresponding range of the main color hues of $Y_{i}$ costumes are concentrated between blue and orange. That is, the costumes of the Yi ethnic group are dominated by warm colors. This result is closely related to the environment in which the Yi people live. The Yi people mostly live in alpine mountains, and the whole nation strongly admires fire culture. The specific embodiment of this cultural pursuit in the color of costumes is that there are more warm colors.

From the perspective of saturation, the results of the two rounds of clustering show that the main color saturation of Yi costumes is almost all below 40, which has a high degree of uniformity, as shown in Figure 7. This reflects the Yi people's thought of pursuing harmonious beauty in costumes. The overall low costume saturation is closely related to the cultural character of the Yi nationality. The Yi ethnic group is a nation with a profound history, having its own writing system thousands of years ago. After thousands of years' development, though diverse in color the costumes are, what the Yi ethnic group pursues is mainly simplicity and generosity. 


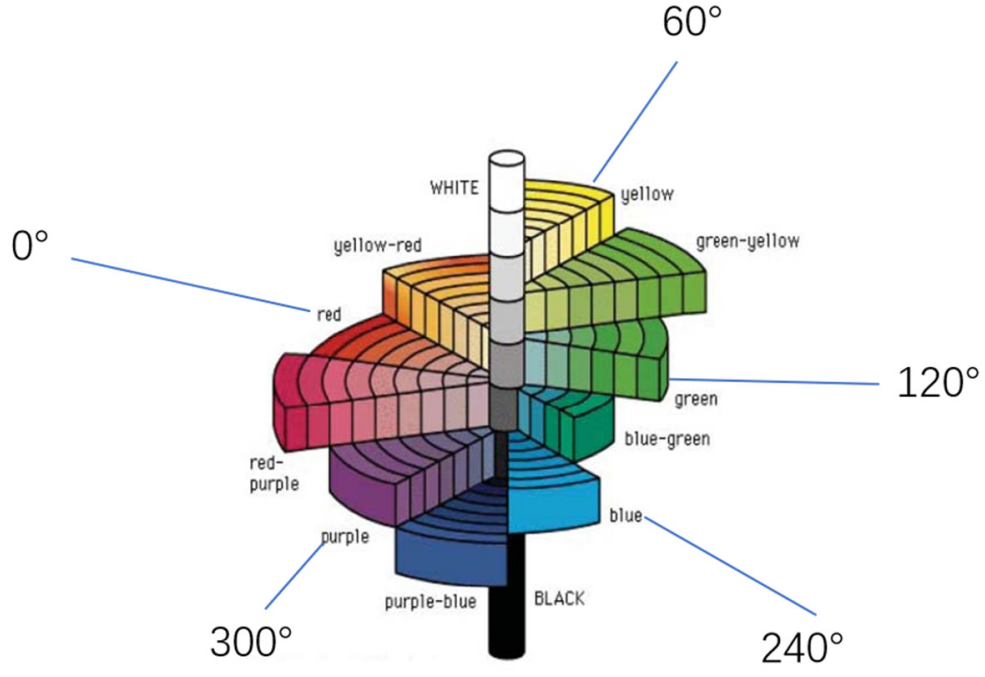

Figure 6. Color ring of HSV.

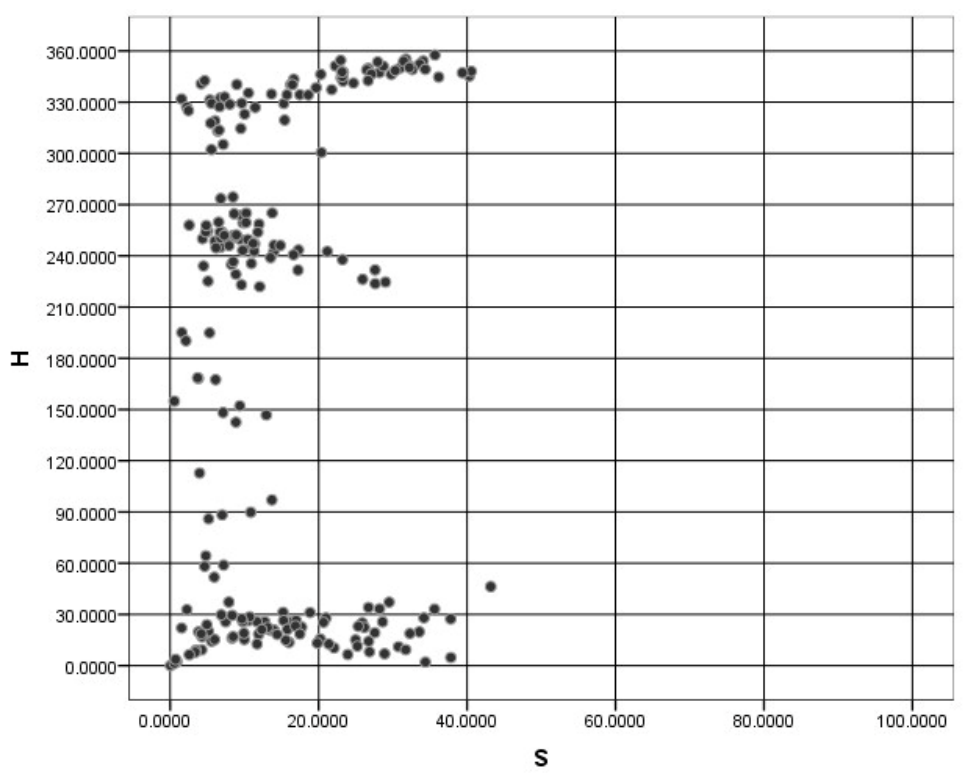

Figure 7. The main color distribution of all clustering results under S-H plane coordinate system.

From the perspective of brightness, the two clustering results show that the main color brightness of Yi costumes is almost all above 40, as shown in Figure 8. The more different the brightness of the color block is, the more different its "weight" is. The smaller the brightness value, the lighter the color; the larger the brightness value, the more stable the color. Seen from the brightness value of the Yi costume colors, the main colors are hardly brisk, and their characteristics are mainly composed of calmness.

The HSV color model separates hue, saturation, and value, which helps to analyze various parameters and their mutual laws. By observing the positions of the results of two rounds of clustering in the $\mathrm{H}-\mathrm{S}, \mathrm{H}-\mathrm{V}$, and $\mathrm{S}-\mathrm{V}$ plane coordinate system, we found that the value of brightness may have a linear correlation to the value of saturation, as shown in Figure 9. 


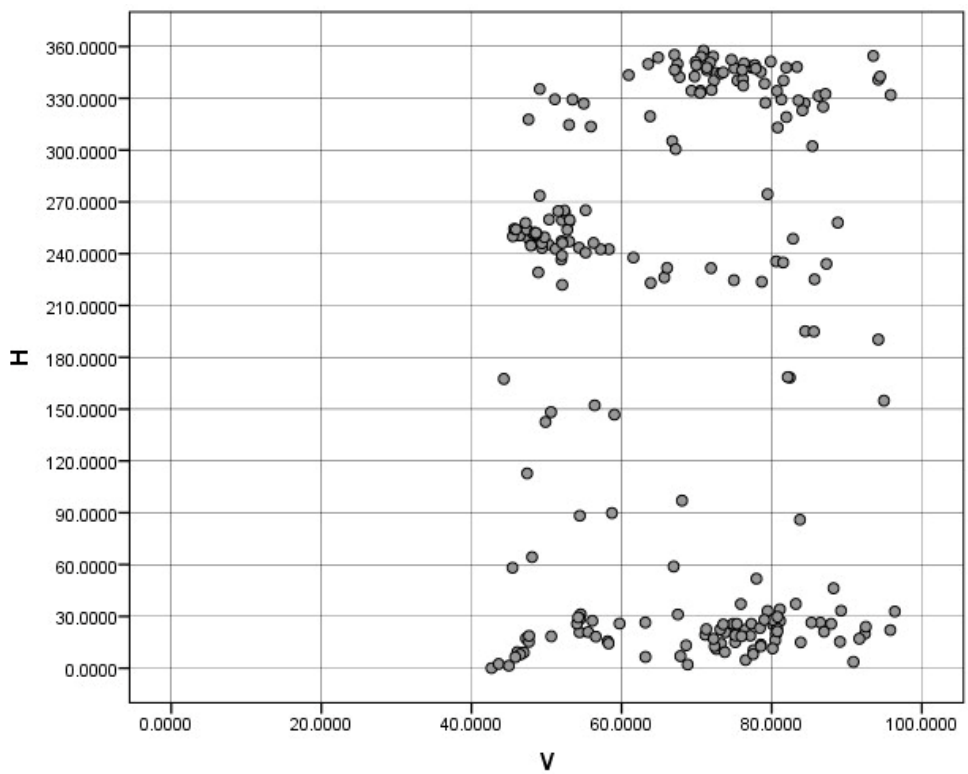

Figure 8. The main color distribution of all clustering results under V-H plane coordinate system.

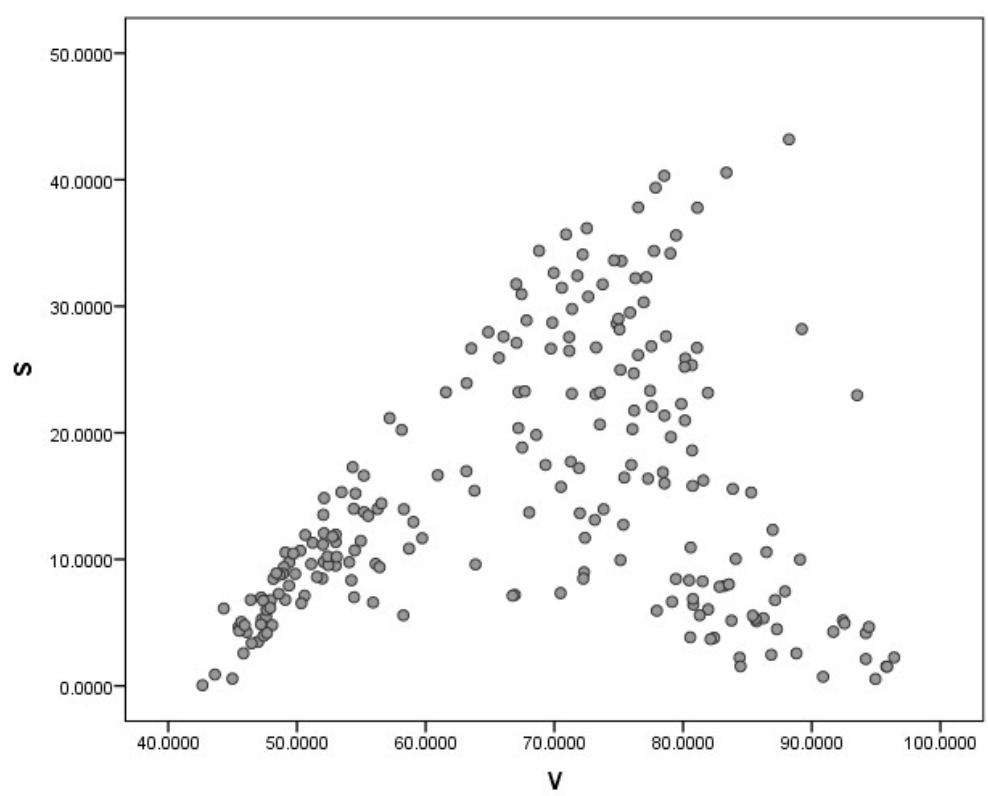

Figure 9. The main color distribution of experimental costumes under S-V plane coordinate system.

We imported the H, S, and V data of the dominant color of Yi costumes obtained by clustering in the experiment into SPSS software. The value of brightness was set as the independent variable, and the value of saturation was set as the dependent variable. Then, the linear regression analysis was performed.

The results show that the significance of the analysis of variance is $0.00<0.05$, indicating that there is a significant linear relationship between the brightness and the color saturation of $\mathrm{Yi}$ costumes. The regression analysis results are good, and the t-test significance level is $0.00<0.05$, indicating that the coefficients of the regression equation are statistically significant. The coefficient of determination is 0.428 , indicating that the explanation degree of brightness for saturation is acceptable. The equation for this regression analysis is:

$$
S=-0.033 V^{2}+4.677 V-141.527
$$


The reason for the linear correlation between saturation and brightness may be related to the age characteristics of the Yi costume color. In the Yi culture, young people wear colorful costumes to attract the opposite sex; the older people wear simple clothes, mainly in cyan and black. Affected by many aspects such as hierarchical view, Yi people may show themselves more by adjusting the brightness and saturation at the same time, when the costume hue frame is relatively stable.

In this part, we found that the distribution of brightness and saturation of the Yi costume color is concentrated, and they are linearly correlated. If the brightness and saturation of the costume color do not conform to the pattern of the clustering results, then the vividness and brightness of the color will be different from the Yi costume color in visual perception, and this difference is expressed in the V-S plane coordinate space as the deviation of the coordinate point of the color from the curve $S=-0.033 V^{2}+4.677 \mathrm{~V}-141.527$. If the $V$ value is the same, the farther the $S$ value is from this curve, the more the color does not conform to the objective law of Yi costume color. Therefore, in this article we take the average distance from the saturation value $S$ of the main colors of the evaluated schemes to the curve $S=-0.033 V^{2}+4.677 \mathrm{~V}-141.527$ in the S-V plane coordinate system (hereinafter referred to as the Distance) as the objective index to evaluate the color schemes of Yi costumes.

\section{Evaluation Method}

In this article, we use the analytical hierarchy process (AHP) to determine the weight of each evaluation index, use the trapezoidal fuzzy number to describe the subjects' score of each subjective index of the evaluated schemes, and use the GRA-TOPSIS method to comprehensively rank the evaluation schemes.

\subsection{AHP Method}

The analytical hierarchy process (AHP) was proposed by American operations research scientists in the 1970s. It is an effective method for solving multiple goals and programs optimization and for judging, evaluating, and making decisions on problems. It has been widely used in recent years in various research areas [43]. Zaman et al. [44] combined the Geographic Information System (GIS) and the AHP to select landfill sites for the government. Muhammad et al. [45] used the AHP and automatic identification system (AIS) to analyze the safety of navigation in the Straits of Malacca in terms of indexes such as hazard scores. Based on customers' emotional preferences, Hadiana et al. [46] developed a helpdesk application using Kansei Engineering and the AHP. The AHP can quantify many evaluation criteria by establishing a multi-level analysis structure model, so as to provide an objective basis for decision-making.

The AHP mainly includes the following steps:

(1) Build a hierarchical model.

(2) Construct a judgment matrix.

(3) Hierarchical ranking and consistency check.

\subsection{GRA-TOPSIS Evaluation Method}

GRA-TOPSIS is a comprehensive evaluation method that combines the technique for order performance by similarity to ideal solution (TOPSIS) and gray correlation analysis (GRA) method. TOPSIS makes decisions based on the Euclidean distance between the evaluation unit and the positive and negative "ideal solutions", and GRA method makes decisions based on the similarity of geometric shapes between the evaluation unit and the positive and negative "ideal solutions" [47]. These two methods have different decisionmaking principles and are highly complementary.

The specific decision-making process of the GRA-TOPSIS comprehensive evaluation method is as follows:

Determine the decision matrix. 
According to the color schemes of Yi costumes to be evaluated, $n$ evaluation indexes are set up to sort and optimize each of the $m$ color schemes. The evaluation values of $m$ alternatives constitute a decision set, denoted as $A=\left\{A_{1}, A_{2}, \cdots, A_{m}\right\}$. Among them, the evaluation value $A_{i}$ of the $i_{\text {th }}$ color alternative corresponding to the evaluation index value under the $k_{t h}$ index is defined as $\omega_{i}(k),(i=1,2, \cdots, m ; k=1,2, \cdots n)$, the decision matrix is defined as $\omega=\left[\omega_{i}(k)\right]_{m \times n}$.

(2) Standardize the decision matrix.

When evaluating the color of Yi costumes, the nature of each evaluation index is different, and there are mainly two evaluation indexes of benefit type and cost type. Benefit-type evaluation indexes have a large-scale nature, and the larger the value, the better; the cost-type evaluation indexes have a small-scale nature, and the smaller the value, the better. In order to eliminate the impact of different dimensions of indexes, cost-type indexes should be firstly forward processed, i.e., the maximum evaluation index value under the $k_{t h}$ index is selected and the evaluation index of each color alternative under the index is subtracted:

$$
x_{i}(k)=\max _{1 \leq j \leq m}\left\{\omega_{j}(k)\right\}-\omega_{i}(k)
$$

After the forward processing, the decision matrix $\omega=\left[\omega_{i}(k)\right]_{m \times n}$ is transformed into a forward decision matrix $X=\left[x_{i}(k)\right]_{m \times n}$. Then, the decision matrix is standardized:

$$
z_{i j}=\frac{x_{i j}}{\sqrt{\sum_{i=1}^{n} x_{i j}^{2}}}
$$

(3) Determine the positive ideal and negative ideal solution.

For a total of $n$ Yi costume color evaluation indexes to be evaluated, the maximum evaluation value corresponding to a total of $m$ schemes constitutes a positive ideal solution, which is defined as $A^{+}$. For the $n$ Yi costume color evaluation indexes to be evaluated, the minimum evaluation value corresponding to $m$ schemes constitutes a negative ideal solution, which is defined as $A^{-}$. After standardizing the decision matrix, the positive ideal and negative ideal solutions are $Z^{+}=\left\{z^{+}(1), z^{+}(2), \cdots, z^{+}(n)\right\}$ and $Z^{-}=\left\{z^{-}(1), z^{-}(2), \cdots, z^{-}(n)\right\}$, respectively, where:

$$
\begin{aligned}
& z^{+}(k)=\max _{1 \leq i \leq m}\left\{z_{i}(k)\right\} \\
& z^{-}(k)=\min _{1 \leq i \leq m}\left\{z_{i}(k)\right\}
\end{aligned}
$$

(4) Calculate the gray correlation degree.

In the $k_{t h}$ index, the correlation coefficient of the positive ideal solution of the $i_{t h} \mathrm{Yi}$ costume color design scheme $A_{i}$ is:

$$
\gamma\left(z^{+}(k), z_{i}(k)\right)=\frac{\min _{i} \min _{k}\left|z^{+}(k)-z_{i}(k)\right|+\rho \max _{i} \max _{k}\left|z^{+}(k)-z_{i}(k)\right|}{\left|z^{+}(k)-z_{i}(k)\right|+\rho \max _{i} \max _{k}\left|z^{+}(k)-z_{i}(k)\right|}
$$

where $\rho$ is the discrimination coefficient, generally $\rho=5$. In the $k_{t h}$ index, the correlation coefficient of the negative ideal solution of the $i_{t h}$ Yi costume color design scheme $A_{i}$ is:

$$
\gamma\left(z^{-}(k), z_{i}(k)\right)=\frac{\min _{i} \min _{k}\left|z^{-}(k)-z_{i}(k)\right|+\rho \max _{i} \max _{k}\left|z^{-}(k)-z_{i}(k)\right|}{\left|z^{-}(k)-z_{i}(k)\right|+\rho \max _{i} \max _{k}\left|z^{-}(k)-z_{i}(k)\right|}
$$


Suppose the index weight vector to be evaluated $\omega=\left(\omega_{1}, \omega_{2}, \cdots, \omega_{n}\right)$, the gray correlation degree of the $i_{\text {th }}$ member $A_{i}$ to the positive ideal solution is:

$$
\gamma\left(Z^{+}, Z_{i}\right)=\sum_{k=1}^{n} \omega_{k} \cdot \gamma\left(z^{+}(k), z_{i}(k)\right)
$$

and the gray correlation degree of the $i_{t h}$ member $A_{i}$ to the negative ideal solution is:

$$
\gamma\left(Z^{-}, Z_{i}\right)=\sum_{k=1}^{n} \omega_{k} \cdot \gamma\left(z^{-}(k), z_{i}(k)\right)
$$

(5) Calculate the Euclidean distance.

The Euclidean distance between the $i_{t h}$ member $A_{i}$ and the positive ideal solution is:

$$
\begin{aligned}
& d\left(Z^{+}, Z_{i}\right)=\sqrt{\sum_{k=1}^{n} \omega_{k} \cdot\left(z^{+}(k)-z_{i}(k)\right)^{2}} \\
& d\left(Z^{-}, Z_{i}\right)=\sqrt{\sum_{k=1}^{n} \omega_{k} \cdot\left(z^{-}(k)-z_{i}(k)\right)^{2}}
\end{aligned}
$$

(6) Integration of gray correlation degree and Euclidean distance.

Normalize $\gamma\left(Z^{+}, Z_{i}\right), \gamma\left(Z^{-}, Z_{i}\right), d\left(Z^{+}, Z_{i}\right)$ and $d\left(Z^{-}, Z_{i}\right)$.

$$
\begin{aligned}
& R\left(Z^{+}, Z_{i}\right)=\frac{\gamma\left(Z^{+}, Z_{i}\right)}{\sqrt{\sum_{k=1}^{n} \gamma\left(Z^{+}, Z_{i}\right)^{2}}} \\
& R\left(Z^{-}, Z_{i}\right)=\frac{\gamma\left(Z^{-}, Z_{i}\right)}{\sqrt{\sum_{k=1}^{n} \gamma\left(Z^{-}, Z_{i}\right)^{2}}} \\
& D\left(Z^{+}, Z_{i}\right)=\frac{d\left(Z^{+}, Z_{i}\right)}{\sqrt{\sum_{k=1}^{n} d\left(Z^{+}, Z_{i}\right)^{2}}} \\
& D\left(Z^{-}, Z_{i}\right)=\frac{d\left(Z^{-}, Z_{i}\right)}{\sqrt{\sum_{k=1}^{n} \gamma\left(Z^{-}, Z_{i}\right)^{2}}}
\end{aligned}
$$

Then, we weight the Euclidean distance and gray correlation degree. When the value of $D\left(Z^{-}, Z_{i}\right)$ and $R\left(Z^{+}, Z_{i}\right)$ are larger, the evaluation scheme tends to be closer to the positive ideal solution. Conversely, when the value of $D\left(Z^{+}, Z_{i}\right)$ and $R\left(Z^{-}, Z_{i}\right)$ are larger, the evaluation scheme tends to be closer to the negative ideal solution. Combining the gray correlation degree and Euclidean distance, we obtain:

$$
\begin{aligned}
& E\left(Z^{+}, Z_{i}\right)=\alpha D\left(Z^{-}, Z_{i}\right)+\beta R\left(Z^{+}, Z_{i}\right) \\
& E\left(Z^{-}, Z_{i}\right)=\alpha D\left(Z^{+}, Z_{i}\right)+\beta R\left(Z^{-}, Z_{i}\right)
\end{aligned}
$$

where $\alpha$ and $\beta$ reflect the decision maker's preference for location and shape, and $\alpha+\beta=1$, $\alpha, \beta \in[0.1]$.

(7) Calculate the relative similarity degree.

The relative similarity degree of the $i_{t h}$ member $A_{i}$ is:

$$
S_{i}=\frac{E\left(Z^{+}, Z_{i}\right)}{E\left(Z^{+}, Z_{i}\right)+E\left(Z^{-}, Z_{i}\right)}
$$

In the formula, the larger the value of $S_{i}$, the greater the scheme is. 


\section{Evaluation Example}

Innovative design of traditional minority costumes has always been a hot spot in the design field. Aiming at this issue, in this article we take the evaluation of the creative design of Yi costume colors as an example to study.

The Yi nationality is a totem-worshiping ethnic group, and Yi people cherish fire. The Torch Festival on June 24 of the lunar calendar is one of their most grand festivals. The worship of fire in Yi ethnic costumes is directly reflected in the use of flame pattern totems. The Yi nationality is also a nature-worshiping ethnic group, the costume patterns of Yi people are varied, and the colors are gorgeous. Yi people incorporate the changing nature of the sun and moon, wind and clouds, the insects, fish, birds, and beasts, and mountains and rivers into clothing patterns to show their style and features [48]. The sun-moon pattern is a pattern abstracted by Yi people based on the sun and the moon, which represents the Yi people's worship of nature and is one of the most representative natural patterns in Yi ethnic costumes.

In terms of pattern layout, the significant feature of Yi ethnic costumes is the continuous change and repetition of patterns. This is in fact a repetitive expression of a single small area, and these small areas are usually arranged neatly and evenly.

Based on the above characteristics, we selected a typical Yi ethnic costume, improved its styles and textures in line with modern aesthetics, and used six different color matching schemes to color it, as shown in Figure 10. The color schemes are divided into three groups, the first group consisting of scheme A and scheme B. We directly extracted the colors from Yi costumes to color them. The second group consists of scheme $C$ and scheme $D$, for which we randomly matched the colors without any reference. The third group consists of scheme E and scheme F, for which we first randomly matched the colors and then adjusted the lightness and saturation of each color according to the curve Equation (8).

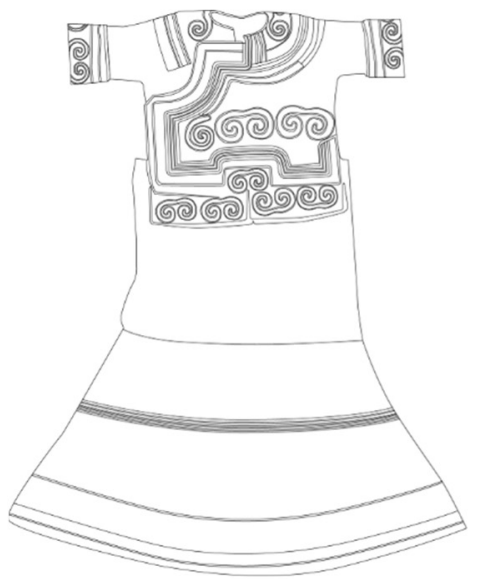

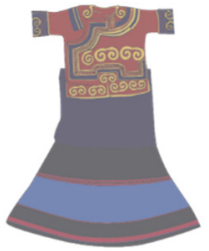

A

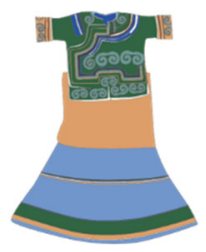

D

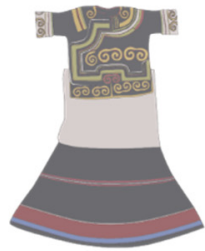

B

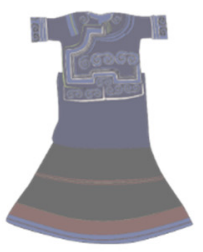

E

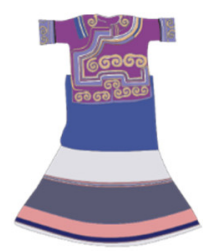

C

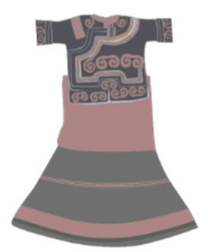

F

Figure 10. Yi costume color design schemes.

\subsection{Index Weight}

The analysis of costume colors as cultural codes can be a code to understand a specific nation and its culture [49]. That is to say, the costume color, as the carrier of culture, should be able to convey the cultural image of the corresponding nation. In addition, the overall feature of Yi ethnic costumes is rich in cultural deposits, simple, practical, and warm [50]. Among these, practical and warm are the functional characteristics, and rich in cultural deposits and simple are the cultural characteristics. Hence, the cultural characteristics of Yi costumes, Simplicity and Richness in cultural deposits are selected as the subjective evaluation indexes for the Yi ethnic costume color schemes. In addition, the Distance index obtained from the K-Means clustering section is used as the objective evaluation index. 
We invited five relevant experts to rank the importance of each index according to the AHP scale table, as shown in Table 2. These experts are two professors who work on folk culture, two costume designers, and one costume collector. After the experts scored, the comparison matrix $R_{1}$ was established, as shown in Table 3.

Table 2. AHP scale table.

\begin{tabular}{ccccccc}
\hline Scale & $\mathbf{1}$ & $\mathbf{3}$ & $\mathbf{5}$ & $\mathbf{7}$ & $\mathbf{9}$ & $\mathbf{2 , 4 , 6 , \mathbf { 8 }}$ \\
\hline Meaning & $\begin{array}{c}\text { Equal } \\
\text { importance }\end{array}$ & $\begin{array}{c}\text { Moderate } \\
\text { importance }\end{array}$ & $\begin{array}{c}\text { Essential } \\
\text { importance }\end{array}$ & $\begin{array}{c}\text { Very vital } \\
\text { importance }\end{array}$ & $\begin{array}{c}\text { Extreme vital } \\
\text { importance }\end{array}$ & $\begin{array}{c}\text { Intermediate value of } \\
\text { neighborhood scales }\end{array}$ \\
\hline
\end{tabular}

Table 3. The comparison matrix $R_{1}$.

\begin{tabular}{cccc}
\hline & Distance & Simplicity & $\begin{array}{c}\text { Richness in Cultural } \\
\text { Deposits }\end{array}$ \\
\hline Distance & 1 & 3 & 5 \\
Simplicity & $1 / 3$ & 1 & 2 \\
$\begin{array}{c}\text { Richness in cultural } \\
\text { deposits }\end{array}$ & $1 / 5$ & $1 / 2$ & 1 \\
\hline
\end{tabular}

That is,

$$
A=\left[\begin{array}{ccc}
1 & 3 & 5 \\
1 / 3 & 1 & 2 \\
1 / 5 & 1 / 2 & 1
\end{array}\right]
$$

Then, according to the method Zuo et al. [51] used, we normalize $A$ twice to obtain its eigenvector $W$.

That is,

$$
W=\left[\begin{array}{c}
3219 / 4968 \\
1142 / 4968 \\
607 / 4968
\end{array}\right]=\left[\begin{array}{c}
0.648 \\
0.230 \\
0.122
\end{array}\right], W=\left(\begin{array}{lll}
0.648 & 0.230 & 0.122
\end{array}\right)^{T}
$$

Next, we calculate the maximum eigenvalue $\lambda_{\max }$ of $A$.

We can obtain:

$$
\begin{gathered}
{\left[\begin{array}{ccc}
1 & 1 & 5 \\
1 / 3 & 1 & 2 \\
1 / 5 & 1 / 2 & 1
\end{array}\right] \times\left[\begin{array}{l}
0.648 \\
0.230 \\
0.122
\end{array}\right]=\left[\begin{array}{l}
1.948 \\
0.690 \\
0.367
\end{array}\right]=\lambda_{\max } W,} \\
\lambda_{\max }=1 / 3 \times(1.948 / 0.648+0.690 / 0.230+0.367 / 0.122)=3 .
\end{gathered}
$$

Then, we calculate $C R$ of $A$. We can obtain $C I=(3-3) /(3-1), C R=\frac{(3-3) /(3-1)}{R I}$ $=0 / 0.52=0<0.1$, i.e., this matrix has an acceptable consistency.

\subsection{Decision Matrix}

A total of fifty subjects were selected to evaluate and score the six color design schemes of the Yi ethnic costume. The gender distribution of these subjects is 23 males (46\%) and 27 females $(54 \%)$; the mean age of these subjects is 23.1 years old $(\mathrm{SD}=0.86)$. The education backgrounds of these subjects are 50 (100\%) with master/doctor education. The trapezoidal fuzzy number was used to represent the evaluation score of each index. Then, the evaluation data of the two subjective indexes, Simplicity and Richness in cultural deposits, were obtained, as shown in Supplementary Tables S1 and S2. In order to understand the relationship between subjective evaluation, objective evaluation, and our proposed evaluation method, we also asked the subjects to additionally rate the degree of similarity between the feelings brought about by the overall color scheme of a sample costume and the feeling brought about by the color scheme of the Yi costume, which we defined as the 
index of Similarity to the Yi costume color scheme, as shown in Supplementary Table S3. Due to the use of trapezoidal fuzzy number scoring, each set of data contains four numbers and is arranged in ascending order from left to right. Therefore, we imported the minimum and maximum values of each set of data into SPSS for reliability and validity analysis, respectively. The results show that the Cronbach's alpha coefficient of the minimum data and maximum data are 0.840 and 0.853 , respectively, both of which are greater than 0.800 , indicating that the reliability of the questionnaire scale is good. The Kaiser-Meyer-Olkin (KMO) test coefficients of the minimum data and maximum data are 0.625 and 0.682 , respectively, both of which are greater than 0.600 , indicating that the validity of the data is acceptable.

The data in Supplementary Tables S1-S3 were de-obfuscated, i.e., the average of each set of data (containing four numbers) was calculated. In addition, the corresponding evaluation data sets were obtained, as shown in Supplementary Tables S4-S6.

According to Equation (8), the evaluation scores of the Distance index were further calculated. Then, the evaluation scores of each index were averaged, and the average value of the six Yi ethnic costume color design schemes on the four evaluation indexes were obtained, as shown in Table 4.

Table 4. Average score of each scheme under the 4 indexes.

\begin{tabular}{cccc}
\hline Schemes & Simplicity & $\begin{array}{c}\text { Richness in } \\
\text { Cultural Deposits }\end{array}$ & $\begin{array}{c}\text { Similarity to Yi } \\
\text { Distance } \\
\text { Costume Color Scheme }\end{array}$ \\
\hline A & $0.5590(0.1698)$ & $0.5612(0.2060)$ & 10.7533 \\
B & $0.6490(0.1620)$ & $0.5445(0.1780)$ & 9.4612 \\
C & $0.4165(0.1944)$ & $0.4540(0.1990)$ & 25.5389 \\
D & $0.4235(0.2247)$ & $0.4145(0.1951)$ & 40.6644 \\
E & $0.6960(0.1549)$ & $0.6750(0.1792)$ & 0.0000 \\
F & $0.6540(0.1389)$ & $0.6510(0.1745)$ & $0.1655)$ \\
\hline
\end{tabular}

Note: Numbers outside the brackets are the mean, and numbers inside the brackets are the standard deviation.

Since the Distance index is a cost-type index, the smaller the data, the better, while the Simplicity and Richness in cultural deposits indexes are both benefit-type indexes, and the larger the data, the better. Therefore, the data of the Distance index are forwarded, as shown in Table 5. The Similarity index to the Yi costume color scheme is set as an overall subjective evaluation index, and we use it to test the proposed evaluation method. Therefore, this index is not included in the GRA-TOPSIS evaluation part.

Table 5. Orthogonalized score of each scheme under the 3 indexes.

\begin{tabular}{cccc}
\hline Schemes & Simplicity & Richness in Cultural Deposits & Distance \\
\hline A & 0.5590 & 0.5612 & 29.9111 \\
B & 0.6490 & 0.5445 & 31.2032 \\
C & 0.4165 & 0.4540 & 15.1255 \\
D & 0.4235 & 0.4145 & 0.0000 \\
E & 0.6960 & 0.6750 & 40.6644 \\
F & 0.6540 & 0.6510 & 40.6644 \\
\hline
\end{tabular}

\subsection{Determine the Positive Ideal and Negative Ideal Solution}

Since the dimension of the Distance index is different from that of the Simplicity and Richness in cultural deposits indexes, according to Equation (10), the decision matrix was standardized, as shown in Table 6. 
Table 6. Standardized score of each scheme under the 3 indexes.

\begin{tabular}{cccc}
\hline Schemes & Simplicity & Richness in Cultural Deposits & Distance \\
\hline A & 0.3954 & 0.4105 & 0.4069 \\
B & 0.4591 & 0.3983 & 0.4245 \\
C & 0.2946 & 0.3321 & 0.2058 \\
D & 0.2996 & 0.3032 & 0.0000 \\
E & 0.4923 & 0.4938 & 0.5532 \\
F & 0.4626 & 0.4762 & 0.5532 \\
\hline
\end{tabular}

We selected positive ideal and negative ideal solutions for all evaluation data sets, and the final positive ideal and negative ideal solutions were obtained:

$$
z^{+}(k)=\{0.4923,0.4938,0.5532\} z^{-}(k)=\{0.2946,0.3032,0\}
$$

\subsection{Calculate the Gray Correlation Degree}

According to Equations (11)-(16), the gray correlation degree between each Yi ethnic costume color design scheme and the positive ideal and negative ideal solutions was calculated. The calculation results are shown in Table 7.

Table 7. Gray correlation degree between color design schemes and ideal solutions.

\begin{tabular}{ccc}
\hline \multirow{2}{*}{ Schemes } & \multicolumn{2}{c}{ Gray Correlation Degree } \\
\cline { 2 - 3 } & Positive Ideal Solution & Negative Ideal Solution \\
\hline A & 0.6880 & 0.5187 \\
B & 0.7383 & 0.4906 \\
C & 0.4984 & 0.7120 \\
D & 0.4238 & 0.9959 \\
E & 1.0000 & 0.4223 \\
F & 0.9704 & 0.4341 \\
\hline
\end{tabular}

\subsection{Calculate the Euclidean Distance}

According to Equations (11), (12), (17) and (18), the Euclidean distance between each Yi ethnic costume color design scheme and the positive ideal and negative ideal solutions was calculated. The calculation results are shown in Table 8.

Table 8. Euclidean distance between color design schemes and ideal solutions.

\begin{tabular}{ccc}
\hline \multirow{2}{*}{ Schemes } & \multicolumn{2}{c}{ Euclidean Distance } \\
\cline { 2 - 3 } & Positive Ideal Solution & Negative Ideal Solution \\
\hline A & 0.1299 & 0.3322 \\
B & 0.1100 & 0.3523 \\
C & 0.3006 & 0.1660 \\
D & 0.4597 & 0.0024 \\
E & 0.0000 & 0.4601 \\
F & 0.0155 & 0.4556 \\
\hline
\end{tabular}

\subsection{Integrate the Gray Correlation Degree and Euclidean Distance}

Since the dimension of the gray correlation degree is different from that of the Euclidean distance, according to Equations (19)-(22), the gray correlation degree and Euclidean distance between each Yi ethnic costume color design scheme and the positive ideal and negative ideal solutions were normalized, as shown in Table 9. 
Table 9. Normalized gray correlation degree and Euclidean distance between color design schemes and ideal solutions.

\begin{tabular}{ccccc}
\hline \multirow{2}{*}{ Schemes } & \multicolumn{2}{c}{ Gray Correlation Degree } & \multicolumn{2}{c}{ Euclidean Distance } \\
\cline { 2 - 5 } & Positive Ideal Solution & Negative Ideal Solution & Positive Ideal Solution & Negative Ideal Solution \\
\hline A & 0.3738 & 0.3366 & 0.2258 & 0.4025 \\
B & 0.4001 & 0.3183 & 0.1912 & 0.4268 \\
C & 0.2708 & 0.4620 & 0.5226 & 0.2011 \\
D & 0.2302 & 0.6462 & 0.7991 & 0.0029 \\
E & 0.5433 & 0.2740 & 0.0000 & 0.5574 \\
F & 0.5272 & 0.2817 & 0.0269 & 0.5520 \\
\hline
\end{tabular}

According to Equations (23) and (24), we defined $\alpha=\beta=0.5$. Then, we integrated the gray correlation degree and Euclidean distance. The results are shown in Table 10. Then, according to Equation (25), we derived the relative similarity degree of the integrated gray correlation and Euclidean distance, as shown in Table 11.

Table 10. Integrated GRA-TOPSIS evaluation score compared with ideal solutions.

\begin{tabular}{ccc}
\hline \multirow{2}{*}{ Schemes } & \multicolumn{2}{c}{ Integrated GRA-TOPSIS } \\
\cline { 2 - 3 } & Positive Ideal Solution & Negative Ideal Solution \\
\hline A & 0.3882 & 0.2812 \\
B & 0.4135 & 0.2548 \\
C & 0.2360 & 0.4923 \\
D & 0.1165 & 0.7227 \\
E & 0.5504 & 0.1370 \\
F & 0.5396 & 0.1543 \\
\hline
\end{tabular}

Table 11. The integrated gray correlation and Euclidean distance relative similarity degree of color design schemes.

\begin{tabular}{cccccc}
\hline $\mathbf{A}$ & B & C & D & E & F \\
\hline 0.5799 & 0.6188 & 0.3240 & 0.1389 & 0.8007 & 0.7776 \\
\hline
\end{tabular}

It can be seen from Table 11 that, according to the GRA-TOPSIS method, the comprehensive ranking result of each scheme is $\mathrm{E}>\mathrm{F}>\mathrm{B}>\mathrm{A}>\mathrm{C}>\mathrm{D}$. It is interesting to note that schemes $\mathrm{E}$ and $\mathrm{F}$, schemes $\mathrm{A}$ and $\mathrm{B}$, and schemes $\mathrm{C}$ and $\mathrm{D}$ are color methods in exactly three different ways. In terms of the final evaluation scores, these three color matching methods also lead to three different evaluation results. Schemes $\mathrm{E}$ and $\mathrm{F}$ perform the best, with corresponding scores of 0.8007 and 0.7776 ; schemes $C$ and D perform the worst, with corresponding scores of only 0.324 and 0.1389 ; schemes A and B are in the middle, with corresponding scores of 0.5799 and 0.6188 . The first group (scheme A and scheme B) uses the original color matching schemes of traditional Yi costumes, while the second group (scheme $\mathrm{C}$ and scheme D) uses random color matching schemes without any references. According to the final evaluation scores, the first group scores significantly higher than the second group, which indicates that our proposed evaluation method can effectively screen color schemes with Yi costume characteristics. According to the final evaluation scores, the third group (scheme E and scheme F) scores higher than the first and second groups, which indicates that the schemes in the third group best fit the characteristics of Yi costumes, and also indicates that by using the schemes of the third group, we may not only be able to design color matching schemes that convey the characteristics of Yi costumes, but also make these schemes more aesthetically pleasing to the public than the traditional color matching schemes of Yi costumes.

In the evaluation of the Simplicity index corresponding to the color design schemes of Yi ethnic costumes, the order of schemes is E $>$ F $>$ B $>$ A $>$ D $>$ C. The scheme E 
performs the best, and the corresponding value is 0.4923 . In addition to scheme $\mathrm{E}$, scheme $\mathrm{F}$ and scheme B also perform well, with corresponding scores of 0.4626 and 0.4591 . In the evaluation of the Richness in cultural deposits index corresponding to the color design schemes of Yi ethnic costumes, the order of the schemes is $\mathrm{E}>\mathrm{F}>\mathrm{A}>\mathrm{B}>\mathrm{C}>\mathrm{D}$. Scheme $A$, with corresponding value of 0.4105 , performs better than scheme $B$. The reason may be that there is a large area of light gray blocks in scheme B, which visually gives subjects a feeling of lightness. In the evaluation of the Distance index corresponding to the color design schemes of Yi ethnic costumes, the order of schemes is $\mathrm{E}=\mathrm{F}>\mathrm{B}>\mathrm{A}>\mathrm{C}>\mathrm{D}$. The corresponding score for scheme $\mathrm{D}$ is zero, which explains why scheme $\mathrm{D}$ is at the bottom of almost all rankings. If it is too far from the curve $S=-0.033 \mathrm{~V}^{2}+4.677 \mathrm{~V}-141.527$ in the $\mathrm{S}-\mathrm{V}$ plane coordinate system, it must be seriously out of line with the traditional $\mathrm{Yi}$ dress color characteristics in terms of visual perception.

In the evaluation of the degree of similarity between the feelings brought about by the overall color scheme of the sample costume and the feeling brought about by the color schemes of the Yi costume, the order of schemes is E $>A>F>B>C>D$. Scheme A performs well in this overall subjective evaluation, with corresponding scores of 0.6950 , 0.5250 points higher than scheme F. This may be the result of the choice of hue. Scheme A and scheme F both use the most classic Yi red, black, and yellow colors, while other color schemes do not have such advantage.

We collated the scores of color schemes in five different evaluation models and then, applying the same method as in Equations (19)-(22), we normalized them and plotted them as line graphs, as shown in Figure 11.

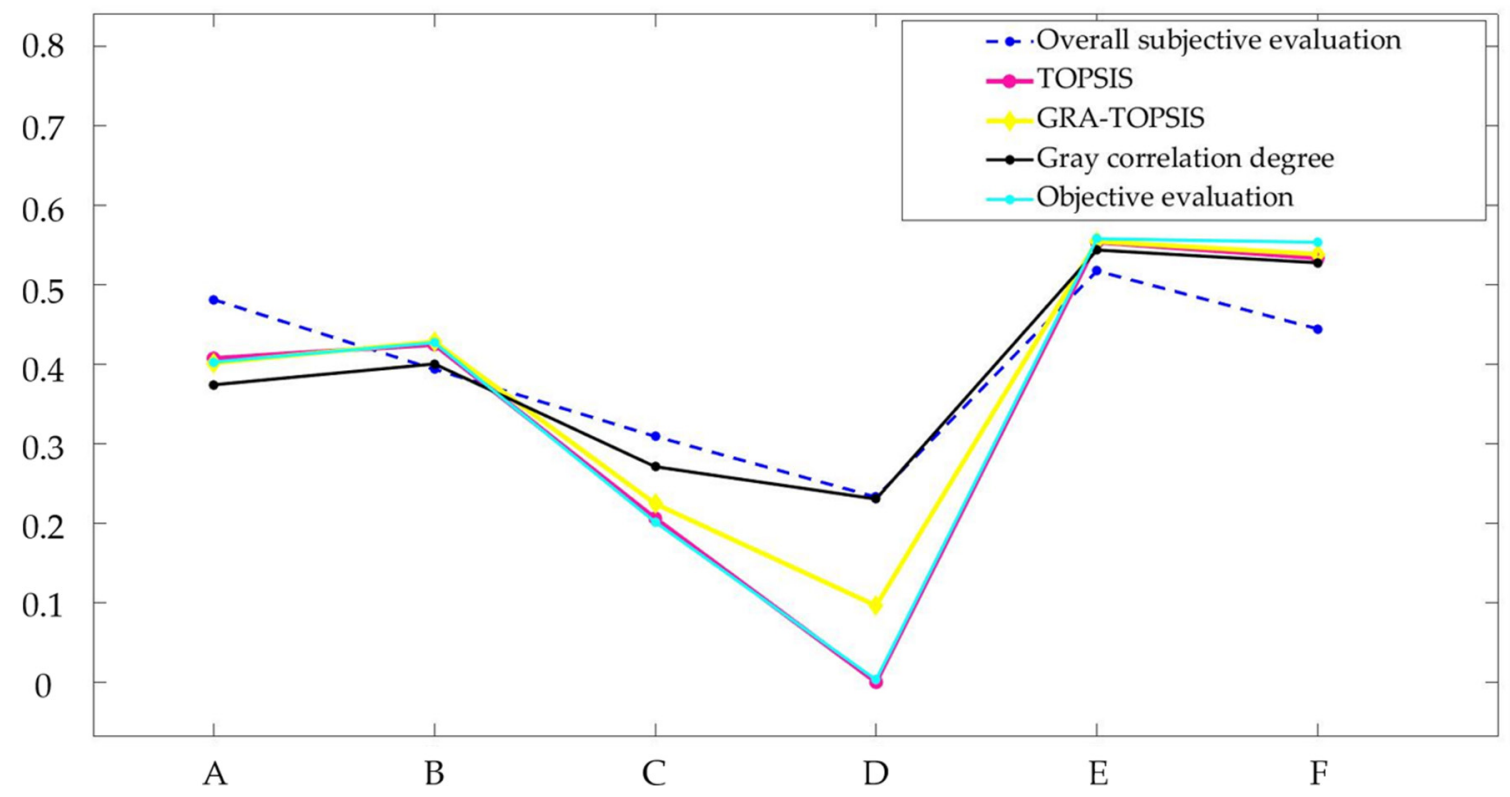

Figure 11. The scores of each color scheme under the five evaluation models.

The blue dotted line represents the scores of each color scheme under the overall subjective evaluation model, i.e., the degree of similarity between the feelings brought about by the overall color scheme of each sample costume and the feeling brought about by the color scheme of the Yi costume. The red solid line represents the scores of each color scheme under the TOPSIS method. The yellow solid line represents the scores of each color scheme under the GRA-TOPSIS evaluation system. The gray solid line represents the scores of each color scheme under the gray correlation analysis method. The cyan solid line represents the scores of each color scheme under the objective evaluation model, i.e., the corresponding scores for each color scheme under the Distance index. The scores of each scheme vary under different evaluation models, but if these evaluation models are 
reasonable, then the lines corresponding to the scores of each color scheme under these evaluation models should have similar fluctuating trends. From Figure 11, on the one hand, we can see that except for the blue dotted line, the fluctuations of other lines tend to be consistent. On the other hand, the objective evaluation scores under the Distance index for scheme $\mathrm{E}$ and scheme $\mathrm{F}$ are identical and no preference can be made from the two. Under the GRA-TOPSIS evaluation system, gray correlation analysis method, and TOPSIS method, the ranking results are all $\mathrm{E}>\mathrm{F}>\mathrm{B}>\mathrm{A}>\mathrm{C}>\mathrm{D}$. Since GRA-TOPSIS can integrate gray correlation degree and Euclidean distance, it can not only evaluate the degree of approximation between the schemes and the ideal situation, but also reflect the pattern of internal changes of each scheme. Using the relative similarity degree as the evaluation criterion, we can more accurately approximate the ideal state. Therefore, we believe that GRA-TOPSIS is the most ideal method for evaluating Yi costume color schemes among the five evaluation models. We can also see from Figure 11 that some schemes score very close to each other under different evaluation models, especially some schemes that score almost exactly the same under TOPSIS and objective evaluation models. We believe that this situation in this paper is coincidental, and if we change the experimental sample, perhaps the lines corresponding to the scores of the color schemes under these evaluation models will still have the same trend, but the situation where the lines almost overlap may no longer occur. We speculate that this situation may be related to the similarity of the metrics adopted by TOPSIS and the objective evaluation model, both of which use distance as a metric. However, this conjecture is subject to further verification.

In the process of evaluating the color schemes of Yi costumes, we found that the results obtained from subjective evaluation alone and objective evaluation alone were contradictory. For instance, when using overall subjective evaluation, the result is $\mathrm{E}>\mathrm{A}>$ $\mathrm{F}>\mathrm{B}>\mathrm{C}>\mathrm{D}$. However, if we use the Distance index for objective evaluation, the result is $\mathrm{E}=\mathrm{F}>\mathrm{B}>\mathrm{A}>\mathrm{C}>\mathrm{D}$. This imbalance between subjective and objective evaluation is likely to lead to wrong decisions. GRA-TOPSIS can combine subjective evaluation with objective evaluation. From Figure 9 we can see that the comprehensive score curve under the GRATOPSIS evaluation system not only conforms to the overall trend of the scoring curves corresponding to the five evaluation methods, but also overcomes the situation of two identical scores in the objective evaluation, which achieves a balance between subjective and objective evaluation, to a certain extent.

\section{Conclusions}

The modern improved design of ethnic costume colors lacks scientific evaluation methods, which may lead to the failure of decision-making. To solve this problem, in this article we proposed a comprehensive evaluation process of ethnic costume color based on the K-Means clustering method. Specifically, in this article we introduced the K-Means clustering method to analyze the color rules of ethnic costumes and converted the corresponding color rules into objective evaluation indexes for the color design of ethnic costumes. Next, the evaluation indexes for the color design of ethnic costumes were raised. Then, the AHP was used to determine the weight vector of the proposed evaluation indexes. Based on the trapezoidal fuzzy number, the users' subjective image perception of the color schemes of ethnic minority costumes was described. Finally, the GRA-TOPSIS evaluation method was used to achieve the ranking of the pros and cons of color design schemes.

The experimental case demonstrates that our proposed method can effectively filter color schemes with minority costume characteristics and can preferentially rank the color schemes to be evaluated according to their relative similarity degree to the color characteristics of minority costumes. K-Means clustering can effectively extract the main colors of minority costumes. In this article, we proposed an objective evaluation index based on the features of the extracted Yi costume main colors in the HSV color space and applied the index to design evaluation within the GRA-TOPSIS framework. We find that the main color features extracted by K-Means clustering can help us to improve the color scheme. 
Specifically, the improved color scheme can be made more consistent with the objective rules of color characteristics of ethnic minority costumes. We will perform further research on the practical application of this method in the future. In this article, we compared the scores of six color schemes under five design evaluation models, and the results show that the ranking of the six color schemes under the subjective evaluation model differs significantly from their ranking under the objective evaluation model. In contrast, our proposed evaluation model based on K-Means clustering uses relative similarity degree as the metric and conforms to the overall score ranking trend of six color schemes under five evaluation models. The method integrates subjective and objective evaluations, overcomes the situation that the results of subjective and objective evaluations contradict each other, and achieves symmetry between the users' subjective evaluations and the objective laws of minority dress colors, to a certain extent.

Supplementary Materials: The following are available online at https:/ / www.mdpi.com/article/10 .3390 /sym13101822/s1, Table S1: Subject's trapezoidal fuzzy scores for color design schemes under the index Simplicity, Table S2: Subject's trapezoidal fuzzy scores for color design schemes under the index Richness in cultural deposits, Table S3: Subject's trapezoidal fuzzy scores for color design schemes under the index Similarity to Yi costume color scheme, Table S4: Subject's average scores for color design schemes under the index Simplicity, Table S5: Subject's average scores for color design schemes under the index Richness in cultural deposits, Table S6: Subject's average scores for color design schemes under the index Similarity to Yi costume color scheme.

Author Contributions: Conceptualization, L.Z.; methodology, L.Z. and Y.Z.; software, L.Z.; validation, L.Z.; formal analysis, L.Z. and Y.Z.; investigation, Y.Z. and D.H.; resources, L.Z.; data curation, L.Z.; writing—original draft preparation, L.Z.; writing—review and editing, L.Z., Y.Z. and D.H.; visualization, L.Z. and D.H.; supervision, Z.W.; project administration, Z.W. All authors have read and agreed to the published version of the manuscript.

Funding: This research received no external funding.

Institutional Review Board Statement: Not applicable.

Informed Consent Statement: Informed consent was obtained from all subjects involved in the study.

Data Availability Statement: The data presented in this study are available in article and Supplementary Material.

Conflicts of Interest: The authors declare no conflict of interest.

\section{References}

1. Shen, X.; Zhou, J.; Xu, T. Minority Costume Image Retrieval by Fusion of Color Histogram and Edge Orientation Histogram. In Proceedings of the Minority Costume Image Retrieval by Fusion of Color Histogram and Edge Orientation Histogram, Okayama, Japan, 26-29 June 2016; IEEE: New York, NY, USA, 2016.

2. Liu, W.L. Research and Innovative Design of Yi Ethnic Costumes in Liangshan, Sichuan. Master's Thesis, Beijing Institute of Fashion Technology, Beijing, China, 2017.

3. Choi, H.H.; Lim, S.A.; HeeKim, J. Promotional Video of Editing Techniques Utilizing Color and Brand Balance. Int. J. Softw. Eng. Appl. 2014, 8, 149-158.

4. Singh, S. Impact of Color on Marketing. Manag. Decis. 2006, 44, 783-789. [CrossRef]

5. He, J.; Chen, D.; Yu, S. Multi-cultural Image Color Design Evaluation Methods for Creative Products of Tourism Culture. Mech. Sci. Tech. Aerosp. Eng. 2021, 40, 92-100.

6. Wang, Y.; Song, F.; Liu, Y.; Li, Y.; Zhang, Z. Color Scheme Compensatory Evaluation Method Based on Eye Movement Tracking Technology. IEEE Access 2020, 8, 214240-214253. [CrossRef]

7. Chen, Y.; Yu, S.; Chu, J.; Yu, M.; Chen, D. Fuzzy Emotional Evaluation of Color Matching for Aircraft Cockpit Design. J. Intell. Fuzzy Syst. 2021, 3, 1-19.

8. Bai, Y.; Xue, Y. Study on Multi-Color Emotion Based on Fashion Color in 2019. Int. J. Cloth. Sci. Tech. 2021, 33, 388-401. [CrossRef]

9. Zhang, X.; Yang, M.; Su, J.; Yang, W.; Qiu, K. Research on Product Color Design Decision Driven by Brand Image. Color Res. Appl. 2020, 45, 1202-1216. [CrossRef]

10. He, J.; Chen, D.; Yu, S. Research on Color Design and Evaluation Method of Cultural Creative Products Based on Color Harmony Theory. J. Northwest. Polytech. Univ. 2020, 38, 766-773. [CrossRef] 
11. Yuan, G.; Xie, Q.; Pan, W. Color Design Based on Kansei Engineering and Interactive Genetic Algorithm. Acad. J. Manuf. Eng. 2017, 15, 12-18.

12. Lu, X.; Xu, J. Color Matching Evaluation of Women's Garments Based on Kansei Engineering. Wool Text. J. 2021, 49, 94-98.

13. Yang, Y.; Chen, D.; Gu, R.; Yu, S. Product Color Design Method Based on Color Case and Grey Relational Analysis. J. Graph. 2016, 37, 509-513.

14. Yang, F.; Ren, J.; Li, J.; Zhang, L. Color Image Design of Mine Excavator Based on Evaluation Method. Packag. Eng. 2017, 38, 246-250.

15. Jiang, Q.; Chen, L.C.; Zhang, J. Perception and Preference Analysis of Fashion Colors: Solid Color Shirts. Sustainability 2019, 11, 2405. [CrossRef]

16. Lin, S.; Hanrahan, P. Modeling How People Extract Color Themes from Images. In Proceedings of the SIGCHI Conference on Human Factors in Computing Systems, Paris, France, 27 April-2 May 2013; Association for Computing Machinery: New York, NY, USA, 2013.

17. Chen, T.; Duan, Y.; Yin, M. Research on HSV Based on Quantification of Apparel Color Features. J. Silk. 2015, 52, $22-26$.

18. Xu, P.; Mao, H.; Zhang, Y.; Gu, B. Study on Analysis Method for Color Composition of Ethnic Costumes. J. Silk. 2019, 56, 24-29.

19. Jain, A.K. Data Clustering: 50 Years Beyond K-means. Pattern Recognit. Lett. 2010, 31, 651-666. [CrossRef]

20. Saxena, A.; Prasad, M.; Gupta, A.; Bharill, N.; Patel, O.P.; Tiwari, A.; Er, M.J.; Ding, W.; Lin, C.T. A Review of Clustering Techniques and Developments. Neurocomputing 2017, 267, 664-681. [CrossRef]

21. Schmid, P. Segmentation of Digitized Dermatoscopic Images by Two-Dimensional Color Clustering. IEEE Trans. Med. Imaging 1999, 18, 164-171. [CrossRef]

22. Lucchese, L.; Mitra, S.K. Unsupervised Low-Frequency Driven Segmentation of Color Images. In Proceedings of the 1999 International Conference on Image Processing (Cat. 99CH36348), Kobe, Japan, 24-28 October 1999; Volume 3.

23. Krishnamachari, S.; Abdel-Mottaleb, M. A Scalable Algorithm for Image Retrieval by Color. In Proceedings of the 1998 International Conference on Image Processing, ICIP98 (Cat. No. 98CB36269), Chicago, IL, USA, 7 October 1998.

24. Li, Z.; Zheng, Y.; Cao, L.; Jiao, L.; Zhong, Y.; Zhang, C.A. Student's T-Based Density Peaks Clustering with Superpixel Segmentation (tDPCSS) Method for Image Color Clustering. Color Res. Appl. 2020, 45, 656-670. [CrossRef]

25. Yang, S.; Li, P.; Wen, H.; Xie, Y.; He, Z. K-Hyperline Clustering-Based Color Image Segmentation Robust to Illumination Changes. Symmetry 2018, 10, 610. [CrossRef]

26. Steinhaus, H. Sur La Division Des Corps Matériels En Parties. Bull. Acad. Polon. Sci. 1956, 1, 801.

27. Lloyd, S. Least Squares Quantization in PCM. IEEE Trans. Inf. Theory 1982, 28, 129-137. [CrossRef]

28. Ball, G.H.; Hall, D.J. ISODATA, A Novel Method of Data Analysis and Pattern Classification; Stanford Research Institute: Menlo Park, CA, USA, 1965.

29. MacQueen, J. Some Methods for Classification and Analysis of Multivariate Observations. In Proceedings of the Fifth Berkeley Symposium on Mathematical Statistics and Probability, Berkeley, UC, USA, 21 June-18 July 1965 and 27 December 1965-7 January 1966; Volume 1, pp. 281-297.

30. Hartigan, J.A.; Wong, M.A. Algorithm AS 136: A K-Means Clustering Algorithm. J. R. Stat. Soc. Ser. C Appl. Stat. 1979, 28, 100-108. [CrossRef]

31. Hansen, P.; Mladenović, N. J-Means: A New Local Search Heuristic for Minimum Sum of Squares Clustering. Pattern Recognit. 2001, 34, 405-413. [CrossRef]

32. Kalczynski, P.; Brimberg, J.; Drezner, Z. Less is More: Simple Algorithms for The Minimum Sum of Squares Clustering problem. IMA J. Manag. Math. 2021. [CrossRef]

33. Liu, X.J.; Cao, Y.J.; Zhao, L.X. Color Networks of Traditional Cultural Patterns and Color Design Aiding Technology. Comput. Integr. Manuf. Syst. 2016, 22, 899-907.

34. Si, Y. Toward the Color Overall Characteristic about Chinese Minority Costumes. Master's Thesis, Zhejiang Sci-Tech University, Hangzhou, China, 2020.

35. Jia, Y.Z.; Jia, J.S. The Beauty of Costume, and the Love for Color: Discussion on Scientific and Artistic of Costume Color Application in Southwest Minority Nationality. Art Des. Res. 2018, 80, 50-53.

36. Zuo, Y. Research on Subjective Evaluation System of Product Design and Its Application-Taking Modern Seat Design as an Example. Master's Thesis, Shandong University, Jinan, China, 2021.

37. Zhang, S.D.; Xue, W.L.; Wei, M.Y.; Zhou, H.L. Application of Eye Tracker in Visual Evaluation of Apparel Fabric Colors. J. Text. Res. 2019, 40, 139-145.

38. Hsiao, S.W.; Yen, C.H.; Lee, C.H.; Chen, R.Q. An Intelligent System for Fashion Colour Prediction Based on Fuzzy C-means and Gray Theory. Color Res. Appl. 2017, 42, 273-285. [CrossRef]

39. Dang, D.; Zeng, P. Target Recognition Based on HSV Color Space and OTSU Algorithm. Intell. Comput. Appl. 2020, 10, 11-16, 22.

40. Midha, S.; Vijay, R.; Kumari, S. Analysis of RGB and YCbCr Color Spaces Using Wavelet Transform. In Proceedings of the 2014 IEEE International Advance Computing Conference (IACC) 2014, Gurgaon, India, 21-22 February 2014; IEEE: New York, NY, USA, 2014.

41. Zhong, S.M.; Zhou, W.L. China's Yi Ethnic Costume; Yun'nan Art Press: Kunming, China, 2006; pp. 1-155.

42. Long, F. Analysis and Application of the Yi People of Color. Master's Thesis, Chongqing University, Chongqing, China, 2012. 
43. Chang, Y.; Liu, B.; Tian, Y. Method and Application of Fuzzy Comprehensive Evaluation of Sweeping Vehicle Modeling Based on AHP. J. Mach. Des. 2017, 34, 121-125.

44. Rahmat, Z.G.; Niri, M.V.; Alavi, N.; Goudarzi, G.; Babaei, A.A.; Baboli, Z.; Hosseinzadeh, M. Landfill Site Selection Using GIS and AHP: A Case Study: Behbahan, Iran. KSCE J. Civ. Eng. 2017, 21, 111-118. [CrossRef]

45. Zaman, M.B.; Kobayashi, E.; Wakabayashi, N.; Pitana, T.; Maimun, A. Implementation of Automatic Identification System (AIS) for Evaluation of Marine Traffic Safety in Strait of Malacca Using Analytic Hierarchy Process (AHP). J. Jpn. Soc. Naval Archit. Ocean Eng. 2012, 16, 141-153.

46. Hadiana, A. Analysis of Customers' Emotional Preferences Using Kansei Engineering and AHP. In Proceedings of the 2018 4th International Conference on Science and Technology (ICST), Yogyakarta, Indonesia, 7-8 August 2018.

47. Chen, Z.; Xu, J. Evaluation of Urban Happiness Index Based on TOPSIS and Grey Correlational Analysis. Statistics Decision. 2021, 37, 59-62. [CrossRef]

48. Zhao, H. Application of Liangshan Yi Patterns in Plane Advertisement Design. Master's Thesis, Southwest Jiaotong University, Chengdu, China, 2013.

49. Lee, J.H.; Kim, Y.I. Analysis of Color Symbology from the Perspective of Cultural Semiotics Focused on Korean Costume Colors According to the Cultural Changes. Color. Res. Appl. 2007, 32, 71-79. [CrossRef]

50. Bao, D. An Analysis of the Color Symbols of Liangshan Yi Nationality. Master's Thesis, Minzu University of China, Beijing, China, 2012.

51. Zuo, Y.; Wang, Z. Subjective Product Evaluation System Based on Kansei Engineering and Analytic Hierarchy Process. Symmetry 2020, 12, 1340. [CrossRef] 